\title{
Sleep Quality Disturbances and Blood Pressure Values
}

\author{
Farský Štefan* and Šidlo Róbert \\ Department of Cardiology, Slovakia
}

*Corresponding author: Farský Štefan, MD, FESC, Dom srdca, Zelená 3, 03608 Martin, Slovakia

\begin{abstract}
The aim of the study was to find out whether early blood pressure (TK) values in patients with fixed and medically treated hypertension correlated with sleep quality during the previous night, and that treatment with rilmenidine affects not only TK but also improves sleep quality by reducing sympathetic activity. In a previous study, 1216 patients with hypertension and metabolic syndrome found that up to $59 \%$ of them reported improvement in sleep during treatment with rilmenidine. We investigated 993 patients with essential hypertension who failed to reach TK target values despite $79 \%$ and $79 \%$ had abdominal obesity. Treatment with rilmenidine was added to the existing treatment. The study lasted for 3 months. At the beginning and end of the study, anthropometric and basic biochemical examinations, repeated heart rate and heart rate measurements over a 10-minute interval, and an assessment of sleep quality according to the Athens Insomnia Scale questionnaire were performed in outpatient clinics.

Following the addition of rilmenidine to the treatment of hypertension, systolic pressure, diastolic pressure, pulse rate and high quality of sleep have significantly decreased $(\mathrm{p}<0.0001)$. Coefficients of correlation between systolic pressure and sleep quality at the start of the study were 0.08 ( $\mathrm{p}<0.0226)$, at the end of the study, $0.14(\mathrm{p}<0.0001)$, between diastolic blood pressure and sleep quality at end of study 0,08 ( $p$ \& lt; 0.015). Coefficients of the correlation between the difference in systolic pressure values and the difference between the results of the baseline and the end of the study were $0.09(p<0.0064)$. In our study, the beneficial effect of rilmenidine in the treatment of hypertense in the sense of a significant decrease in heart rate, heart rate retardation and improvement of sleep quality as assessed by a standardized questionnaire was confirmed. In addition, we found interesting connections between sleep quality and systolic and diastolic blood pressure values before and during treatment with rilmenidine. These results are in line with current literature that has shown that reduced range and reduced sleep quality adversely affect TK, hypertension, and overall cardiovascular risk in both adolescent and adult hypertensive patients.
\end{abstract}

Keywords: Sleep; Sleep Quality; Blood Pressure; Hypertension; Rilmenidine

\section{Introduction}

When high blood pressure (TK) values are high, doctors ask their patients the question: "Are you sleeping well?" What is the relationship between hypertension and sleep disorders? Is high blood pressure one of the causes of sleep disorders or are sleep disturbances causing the onset of TK the next day, possibly even for a longer period of time? Now we do not consider acute, temporary sleep disorders under the influence of current stress states that clearly increase the "drive" of sympathy. We will also not deal with

patients with sleep apnea syndrome in whom this relationship is clearly demonstrated. We think of chronic insomnia, which often has no immediate inducing factor in its history. In the "over DRIVE" program, we attempted to ascertain whether early TK values in patients with fixed and medically treated hypertension correlated with sleep quality during the previous night, and whether treatment with rilmenidine would favorably affect not only TK but also improved quality by reduced sympathetic "overdrive" sleep. Already in SYMPATHY [1], we are on sample 1216 hypertensive patients and metabolic syndrome have found that up to $59 \%$ of them reported improvement in sleep during rilmenidine treatment.

\section{Material and Methods}

We investigated 993 patients, of whom $38.6 \%$ were male and $61.4 \%$ female, aged $23-92$, mean age $61.24+-11.32$ years (Table 1 ). The average weight was $86.63+-15.02 \mathrm{~kg}$, average BMI $30.27+-5.12$ $\mathrm{kg} / \mathrm{m}^{2}$, average waist circumference $98.24+-13.5 \mathrm{~cm}$. 
Table 1: Basic descriptive statistics of the connected variables.

\begin{tabular}{|c|c|c|c|c|c|c|c|}
\hline & $\mathrm{N}$ & Mean & $\begin{array}{l}\text { St. } \\
\text { Dev }\end{array}$ & \multicolumn{2}{|c|}{$95 \%$ CI } & Min & Max \\
\hline Age & 972 & 61,24 & 11,32 & 60,52 & 61,95 & 23 & 92 \\
\hline $\begin{array}{c}\mathrm{M} 0_{-} \\
\text {weight }\end{array}$ & 993 & 86,63 & 15,02 & 85,69 & 87,57 & 44 & 156 \\
\hline $\begin{array}{l}\mathrm{M}_{-} \\
\text {height }\end{array}$ & 993 & 168,48 & 8,48 & 167,95 & 169,01 & 145 & 195 \\
\hline $\begin{array}{c}\text { M0_ } \\
\text { waist }\end{array}$ & 969 & 98,24 & 13,53 & 97,39 & 99,09 & 58 & 152 \\
\hline $\begin{array}{l}\mathrm{M} 0_{-} \\
\mathrm{BMI}\end{array}$ & 899 & 30,27 & 5,12 & 29,93 & 30,60 & 17 & 58 \\
\hline $\begin{array}{c}\text { M0_ } \\
\text { BMI_V }\end{array}$ & 993 & 30,53 & 4,98 & 30,22 & 30,84 & 16,22 & 56,61 \\
\hline $\begin{array}{l}\text { M0_ } \\
\text { sBP_1 }\end{array}$ & 981 & 160,01 & 14,48 & 159,11 & 160,92 & 120 & 210 \\
\hline $\begin{array}{c}\text { M0_ } \\
\text { sBP_2 }\end{array}$ & 969 & 156,73 & 14,43 & 155,82 & 157,64 & 105 & 210 \\
\hline $\begin{array}{l}\text { M0_ } \\
\text { sBP_A }\end{array}$ & 877 & 158,12 & 14,13 & 157,19 & 159,06 & 125 & 208 \\
\hline $\begin{array}{c}\text { M0_ } \\
\text { dBP_1 }\end{array}$ & 981 & 95,32 & 8,58 & 94,79 & 95,86 & 60 & 130 \\
\hline $\begin{array}{c}\text { M0_ } \\
\text { dBP_2 }\end{array}$ & 969 & 93,17 & 8,46 & 92,64 & 93,70 & 60 & 120 \\
\hline $\begin{array}{c}\text { M0 } \\
\text { dBP_A }\end{array}$ & 878 & 94,02 & 8,19 & 93,48 & 94,56 & 65 & 125 \\
\hline $\begin{array}{c}\text { M0_ } \\
\text { HR_1 }\end{array}$ & 982 & 77,40 & 10,57 & 76,73 & 78,06 & 49 & 142 \\
\hline $\begin{array}{c}\text { M0_ } \\
\text { HR_2 }\end{array}$ & 967 & 75,95 & 10,07 & 75,31 & 76,58 & 49 & 138 \\
\hline $\begin{array}{c}\text { M0_ } \\
\text { HR_A }\end{array}$ & 873 & 76,07 & 10,13 & 75,40 & 76,74 & 49 & 140 \\
\hline $\begin{array}{c}\mathrm{DO}_{-} \\
\text {points }\end{array}$ & 993 & 13,49 & 7,48 & 13,03 & 13,96 & 0 & 39 \\
\hline
\end{tabular}

\section{Criteria for Inclusion}

a) Over 18 years of age, essential hypertension.

b) Uncontrolled TK in the treatment of hypertension by combining 2 or more drugs.

\section{Exclusion Criteria}

a) Patients are systematically treated with psychopharmaceuticals, hypnotics.

b) Patients who were contraindicated with rilmenidine.
The patients were in the care of the specialists-internists who carried out all the examinations in their internal ambulances. The list of participating internists is attached.

\section{Schedule}

M0-inclusion of the patient.

M1-control visit after 1-month.

M3 - Final visit after 3 months from the beginning of the program.

M0

a) Patient selection and inclusion according to selection and exclusion criteria.

b) anthropometric examination.

c) Measuring heart rate and measuring heart rate (2 times in succession over 10 minutes).

d) Quality assessment of sleep via questionnaire (Table 2).

Table 2.

\begin{tabular}{|c|c|c|c|c|}
\hline & $\begin{array}{l}\mathrm{Nev} \\
\text { er }\end{array}$ & $\begin{array}{l}\text { Som } \\
\text { et }\end{array}$ & $\begin{array}{l}\text { Oft } \\
\text { en }\end{array}$ & $\begin{array}{l}\mathrm{A} 1 \\
\mathrm{w}\end{array}$ \\
\hline 1. I have a problem with falling asleep & \multicolumn{4}{|l|}{$r$} \\
\hline 2. It takes me more than an hour to fall asleep & \multicolumn{4}{|l|}{$r$} \\
\hline 3. I wake up more than 3 times at night & \multicolumn{4}{|l|}{$r$} \\
\hline 4. It takes me long time to fall asleep again after waking up & \multicolumn{4}{|l|}{$r$} \\
\hline 5. I am waking up early in the morning & \multicolumn{4}{|l|}{$r$} \\
\hline 6. I'm worried that I won't sleep & \multicolumn{4}{|l|}{$r$} \\
\hline 7. I am drinking alcohol in the evening to fall asleep easier & \multicolumn{4}{|l|}{$r$} \\
\hline 8. When I am lying I feel my feet restless and I have unpleasant & \multicolumn{4}{|l|}{$r$} \\
\hline 9. I have a problem to get up in the morning & \multicolumn{4}{|l|}{$r$} \\
\hline 10. I feel tired in the morning & \multicolumn{4}{|l|}{$r$} \\
\hline 11. Sleeping does't bring me refreshment & \multicolumn{4}{|l|}{$r$} \\
\hline 12. My sleep is short even though I am in bed long enough & \multicolumn{4}{|l|}{$r$} \\
\hline 13. I feel tired whole day since morni & \multicolumn{4}{|l|}{$r$} \\
\hline
\end{tabular}

Evaluation: Never 0 points

Sometimes 1 point

Often 2 points

Always 3 points

Number of reached points:

Total score $<10$ : no sleeping disorder

Total score $>11$ : suspicion of insomnia

Total score $>25$ : insomnia

e) Detection of the presence of associated cardiovascular risk factors. 
f) Optimization of hypertension therapy by adding rilmenidine $1 \mathrm{mg}$ tbl. per os 1 times a day.

\section{M1}

a) Measuring heart rate heart rate (2 times in 10 minutes increments).

b) Dose adjustment of rilmenidine, systolic TK> $140 \mathrm{mmHg}$ and / or diastolic TK> $90 \mathrm{mmHg}$

\section{M3}

a) Anthropometric examination.

b) Measurement of heart rate and heart rate (2 times in a 10-minute interval).

c) Quality assessment of sleep via questionnaire (Table 2).

\section{Results}

In Table 1 the basic characteristics of the set at time M0, including the values of systolic and diastolic blood pressure, heart rate and point values of the questionnaire at the beginning of the study [2]. It is evident that the mean values of systolic and diastolic blood pressure were slightly increased and mean BMI values were at the beginning of obesity. The average score values of the questionnaire were in the "suspected insomnia" band. Three months of added rilmenidine treatment, the quality of sleep improved, and the mean value of the questionnaire score was in the "no sleep disorder" band (Figure 1). At the same time, the mean values of both systolic and diastolic blood pressure, which have reached the controlled hypertensive band, decreased markedly, statistically significant. The mean heart rate decreased by 6 strokes / min, similarly to our previous studies with rilmenidine (Figure 2).

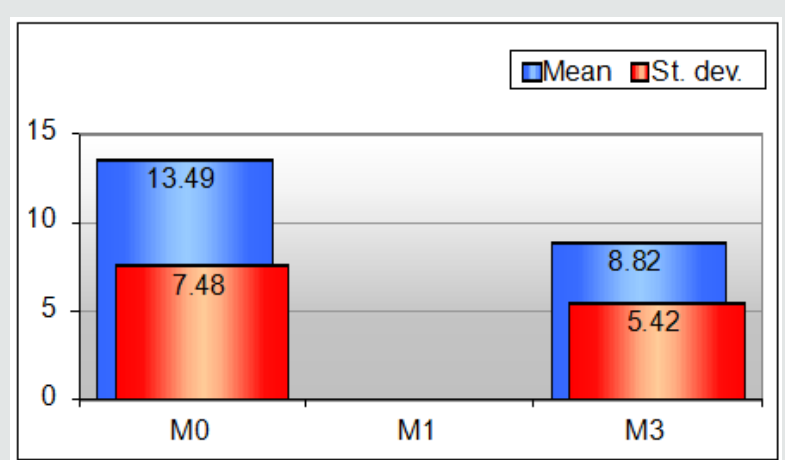

Figure 1: Changes of average sleeping quality total score values at $M 0$ versus $M 3$ visits.

By correlation analysis between the sleep quality questionnaire scores and the blood pressure values we found some dependence on blood systolic blood pressure at the beginning of the study (slightly above the statistical significance limit), more significant dependence was found for both systolic and diastolic TK at the end of the study (Figure $3 \& 4$ ). Statistically significant correlations of blood systolic blood pressure differences were also statistically significant, and the difference between the point score at the start and end of the study (delta sTK versus the delta score) (Table 3).
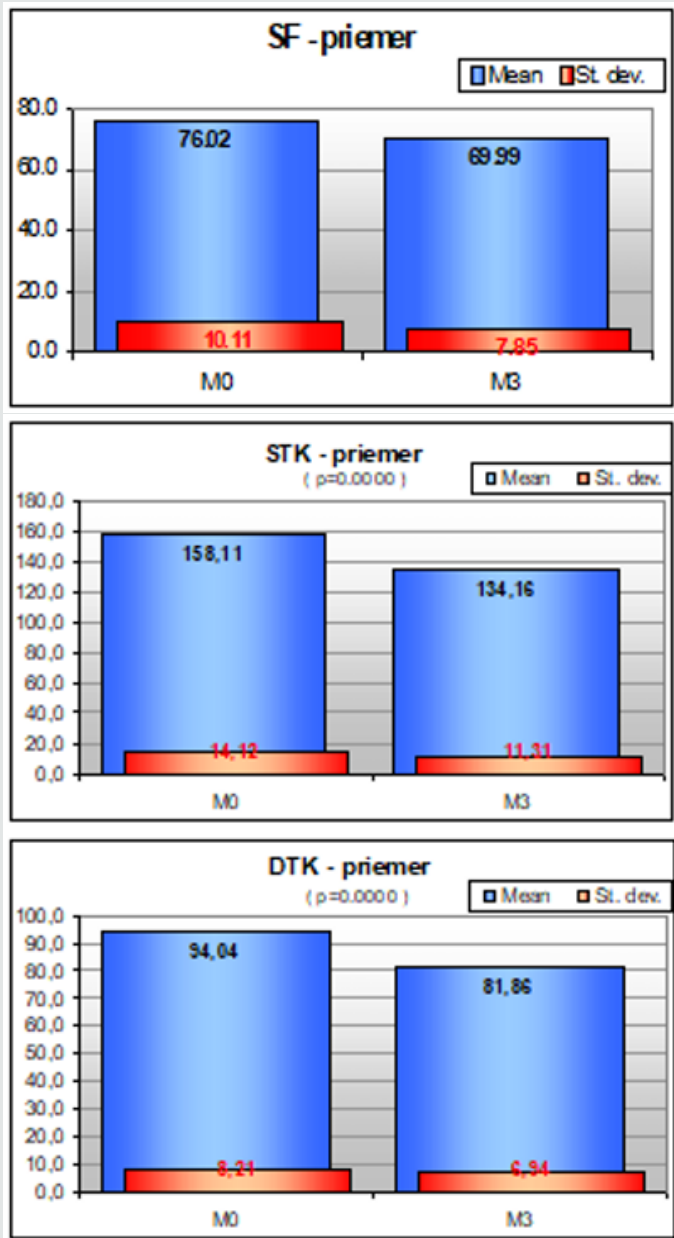

Figure 2: Changes in average values of $\mathrm{sBP}, \mathrm{dBP}$ and $\mathrm{HR}$ from M0 to M3.

$(\mathrm{STK}-$ priemer $=\mathrm{sBP}$ average, $\mathrm{DTK}$ priemer $-\mathrm{dBP}$ average, SF priemer $=H R$ average) .

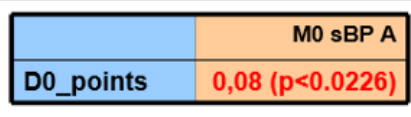

M0_sBP vs. D0_points

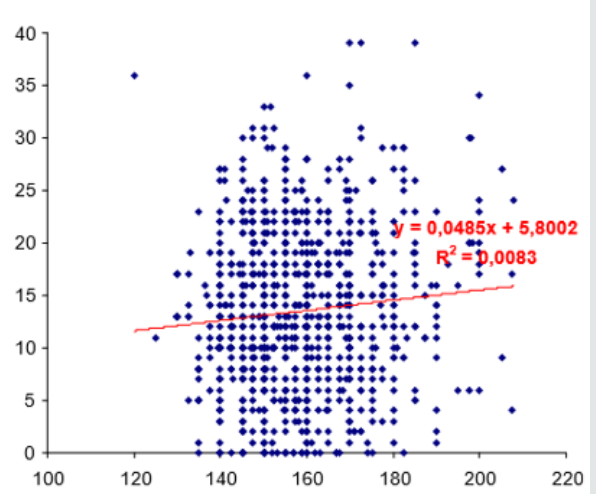

Figure 3: Ratios of correlation between average values of $\mathrm{sBP}$ and results of questionnary at visit M0. 


\begin{tabular}{|l|l|l|}
\hline & M3 sBP A & M3 dBP A \\
\hline D3_points & $0.14(p<0.0001)$ & $0.08(p<0.015)$ \\
\hline \multicolumn{3}{|c|}{ M3_sBP vs. D3 points }
\end{tabular}

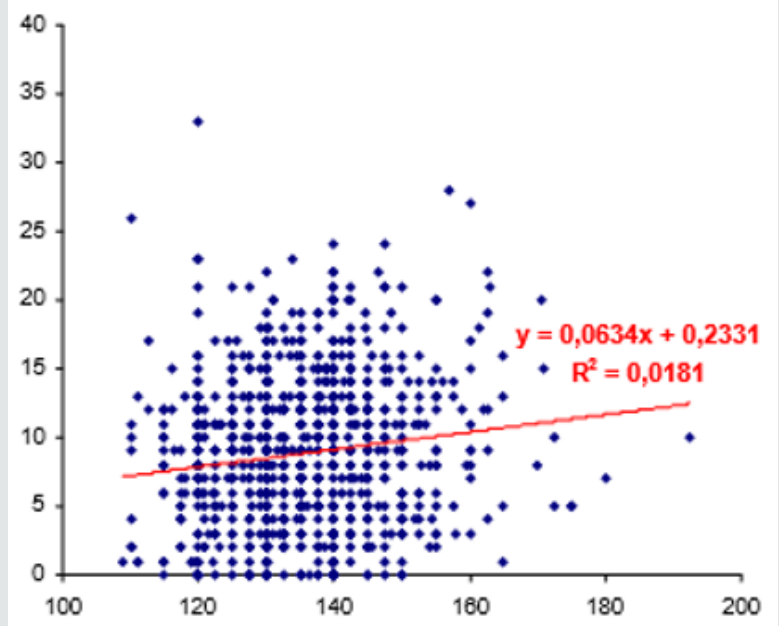

Figure 4: Ratios of correlation between average values of $\mathrm{BP}$ and results of questionnary at visit M3.

Table 3: Correlations between changes in SBP and dBP values versus changes of total sleeping score values (DTK $=\mathrm{dBP}$ ).

\begin{tabular}{|c|c|c|}
\hline & sBP_M0 - sBP_M3 & DTK_M0 - DTK_M3 \\
\hline DD-D3 body & $-0.09(\mathrm{p}<0.0064)$ & -0.07 \\
\hline
\end{tabular}

\section{Discussion}

After looking at the possible pathophysiological mechanisms between abdominal obesity, insulin resistance, and hypertension, we have now paid attention to a new topic - looking for possible links between sleep and hypertension. Both phenomena have a high prevalence in the population. Hypertension and "prehypertension" occur in about $2 / 3$ of the adult population of Slovakia, with the prevalence rising significantly with increasing age [3]. Insomnia affects about $20 \%$ (4-48\%) of the population, its prevalence also increases with age [4]. The common denominator of both disorders is probably the wrong way of life and the accumulation of stress effects with excessive sympathetic activation. In the treatment, the overpotential potential of rilmenidine-type central sympatholytic is present. But proving the interdependence in terms of evidencebased medicine is not at all easy. If a certain relationship exists, is the primary sleep disorder with a subsequent increase in TK, or does the patient sleep poorly because of high TK? On the other hand, in patients with sleep apnea syndrome, this relationship is to a large extent proven and the primary cause is likely to be breathing disorders during sleep $[5,6]$.

The diagnosis of insomnia itself is largely based on the reproduction of the subjective feelings of the patient (questionnaires), less on objective measurements (actimetry, polysomnography) which are almost inaccessible for the diagnosis of insomnia, respectively. focused on the diagnosis of sleep apnea syndrome.

Insomnia is defined by the following symptoms:

a) Sleeping takes $>30$ minutes.

b) Re-sleep after waking at night lasts $>30$ minutes.

c) Wake up $<30$ minutes before the scheduled time.

d) Sleep disorders occur $>3$ times a week.

e) Duration of malfunctions $>$ one month.

f) Sleep efficiency $<85 \%$ (Sleep efficiency is the time of sleep in relation to the time spent in bed for sleeping).

During insomnia, sleep disorders of NREM I, NREM IV (delta), sleep architecture are impaired, individual stages are changed [7]. Insomnia is more susceptible to individuals with certain predispositions and psychophysiological manifestations, e.g. with an increased response to grief, reduced calming capacity, with a tendency to rumination. Short sleep and sleep disturbances are a risk factor for hypertension, and good sleep is associated with a decrease in TK values [8]. In so-called. CARDIA sleep study in 578 adults repeatedly tested over 3 years with 3-meter acclimetry for 3 consecutive nights showed that reducing sleep duration by 1 hour was associated with an increased risk of hypertension by $37 \%$. Javaheri et al. [9] investigated 238 adolescents in the sleeping lab and the actimetry,

Who simultaneously measured TK for two days 9 times. With a shorter sleep duration of 6.5 hours, the risk of "prehypertension" increased 2.8-fold and when sleep-efficiency was <85\% 4.5-fold. After adjusting for the presence of other potentially effective factors, these values were 2.5 resp. 3.5 [9]. In the sub-group with sleep disorders, the systolic TK values were on average $4 \mathrm{mmHg}$ higher than in the subgroup without these disorders. The short duration of sleep is associated with a rise in nighttime sleep up to 4.4 times the normal duration of sleep [10], while the overall cardiovascular risk is also significantly increased. According to Reuters Health, it is estimated that $30-40 \%$ of the population sleeps less than 5 hours in the United States and the United Kingdom, resulting in an enormous range of possible impacts on the health of the population. In addition, short duration and sleep disturbances are accompanied by increased daily drowsiness and increased risk of injuries, car crashes and bad decisions. Daily sleepiness also significantly increases cardiovascular mortality in older people. In a study of three French cities, Empana et al. [11], daily sleepiness was questioned by a structured interview on a sample of 9300 people aged $>65$ years. They found that in daytime sleepiness (even after adjusting for age, gender, BMI and disease present) cardiovascular mortality increased by $49 \%$, overall mortality by $33 \%$, and saxacological mortality did not increase [11]. On the contrary, in a 
great Greek study, the prosperity of the afternoon sleep with short sleep, siesta [12], proved to be beneficial. In observance of the siosy, even after adjusting for possible distortive factors, the mortality rate for ischemic heart disease was reduced by $37 \%$.

Certain sleep times and adequate quality are important conditions for the proper functioning of the immune system and glucose metabolism. Short sleep duration is associated with an increased risk of obesity and diabetes, which is explained by a hormone-induced increased appetite and appetite for sweet meals and reduced energy expenditure throughout the day. At a minimum, 7 hours of sleep is generally considered. Some say they feel well after a short sleep, but testing has shown that their performance is not optimal [8]. The hyperactivity of the sympathetic nervous system is considered to be the main mechanism for increasing TK in chronic insomnia. Night "micro" wake increases sympathetic activity and thus increases TK. Another possible mechanism is an aging-induced decrease in melatonin level, which not only synchronizes the circadian rhythm of sleep and wakefulness but reduces TK at nighttime by its vasodilating action. High TK values at night, Non-dipping hypertension in patients with sleep disorders is 3 times more common than those who sleep well [13]. This type of hypertension is associated with high cardiovascular risk and nightly TK values are the strongest prognostic factor [14].

Our results are consistent with current literature. We have confirmed that there is a linear relationship between sleep quality expressed by point scores and systolic TK values in patients with essential hypertension. This relationship, expressed by the correlation and determination coefficients, was statistically even more potent after the addition of rilmenidine to the treatment of hypertension, and after three months of treatment, the relationship between sleep quality and diastolic TK was also statistically significant. Rilmenidine is a drug that, by its central action, reduces sympathetic hyperactivity and thus affects not only elevated TK but also the quality of sleep. Which of these mechanisms of action was a priority, therefore, it was first a decrease in TK and consequently a better sleep or a better sleep and therefore decreased TK, it is not possible to determine our results. It is highly probable that both mechanisms would overlap and result in improved health. Clinical experience has shown that rilmenidine is particularly effective in the treatment of hypertension in patients with explosive, hyperactive, brisk reactions. Certain limitations in our program have been used for screening methods when subjective patient data was used to assess sleep quality and TK was measured by the standard clinical method used in the outpatient clinic. Higher validity of results can be expected when to use more objective and reproducible methods of sleep quality assessment, such as actimetry, polysomnography, outpatient 24-hour TK monitoring.

\section{Conclusion}

It should be emphasized that physicians should also take into account the quality and duration of sleep in the care of patients with essential hypertension and in assessing cardiovascular risk. In selecting treatment, consideration should also be given to the possibility of positively influencing the quality of sleep and nighttime TK values, not just a decrease in TK during the day. In the prevention of hypertension, it is necessary to put emphasis not only on optimal weight, regular exercise, but also on quality and long enough sleep.

\section{References}

1. Farský Š Sympathy (2007) Study: Sympathetic hyperactivity in the pathogenesis of hypertension in metabolic syndrome. Medical Leaves 45: 13-14.

2. Soldatos CR, Dikeos DG, Paparrigopoulos TJ (2000) Athens Insomnia Scale. J Psychosom Res 48(6): 555-60.

3. Barakova A, Avdicová M, Vallová Z (2004) The prevalence of hypertension in the SR population and the extent of its impact on selected indicators of health statistics. Cardiol 13(3): 63-70.

4. Ohayon MM (2002) Epidemiology of insomnia: What we know and what we still need to learn. Sleep Med Rev 6(2): 97-111.

5. Peppard PE, Young T, Palta M (2000) Prospective study of the association between sleep-disordered breathing and hypertension. N Enlg J Med 342: 1378-84.

6. Baguet JP, Narkiewicz K, Mallion JM (2006) Update on hypertension management: obstructive sleep apnea and hypertension. J Hypert 24(1): 205-208.

7. Tomori Z, Redhammer R, Donic V (1999) Fundamentals of Sleep Medicine. Military Aviation Academy Košice pp. 368.

8. Knutson KL, Van Cauter E, Rathouz PJ (2009) Association between sleep and blood pressure in midlife. The CARDIA sleep study. Arch Intern Med 169(11): 1055-1061.

9. Javaheri S, Storfer Isser A, Rosen CL (2008) Sleep quality and elevated blood pressure in adolescents. Circulation 118(10): 1034-1040.

10. Eguchi K, Pickering T, Schwartz JE (2008) Short sleep duration as an independent predictor of cardiovascular events in Japanese patients with hypertension. Arch Intern Med 168(20): 2225-2231.

11.Empana JP, Dauviliers Y, Dartigues JF (2009) Excessive daytime sleepiness is an independent risk indicator for cardiovascular mortality in community dwelling elderly. The Three Cities Study. Stroke 40(4): 1-6.

12. Naska A, Oikonomou E, Trichopola A (2007) Siesta in healthy adults and coronary mortality in the general population. Arch Intern Med 167(3): 296-301.

13. Yilmaz MB, Yalta K, Turgut $O 0$ (2007) Sleep quality among relatively young patients with initial diagnosis of hypertension: dippers vesus non-dippers. Blood Press 16(2): 101-105.

14. Dolan E, Stanton A, Thijs L (2005) Superiority of ambulatory over clinical blood pressure measurement in predicting mortality. The Dublin Outcome Study. Hypertension 46: 156-161. 
(C) Co This work is licensed under Creative

To Submit Your Article Click Here: Submit Article

DOI: 10.32474/ACR.2018.01.000120

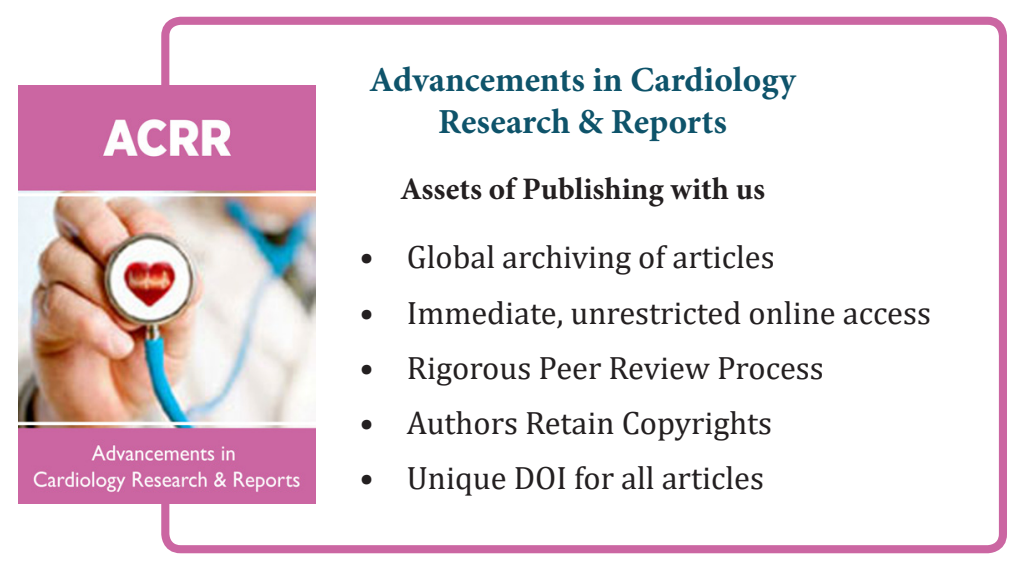

